\title{
An Efficient Design of Security Accelerator for IEEE 802.15.4 Wireless Senor Networks
}

\author{
Ohyoung Song and Jiho Kim \\ School of Electrical and Electronic Engineering, Chung-Ang University, \\ 221, HukSuk-Dong, DongJak-Gu, Seoul, Korea \\ song@cau.ac.kr, jihokim@wm.cau.ac.kr
}

\begin{abstract}
In this paper, we provide a low cost AES core for ZigBee devices which accelerates the computation of AES algorithms. Also, by embedding the AES core, we present an efficient architecture of security accelerator satisfying the IEEE 802.15.4 specifications. In our experiments, we observed that the AES core and the security accelerator use fewer logic gates and consume lower power than other architectures based on blockwide and folded ones.
\end{abstract}

Keywords-WPAN, ZigBee security, Secure sensor network

\section{INTRODUCTION}

The cryptographic operation in wireless device using small memory and low power processor causes the system overhead, so that it decreases the performance of other tasks. Therefore, it is necessary to implement the security hardware that is dedicated to the cryptographic operation [1].

There are many implementation results for AES (Advanced Encryption Standard) design on FPGA or ASIC. Early AES designs were mostly straightforward implementations of various loop unrolled and pipelined architecture [2-6] with limited number of architectural optimizations, which resulted in poor resource utilization. Later FPGA implementations focused on better utilization of FPGA resources [7-10].

Recent research focused on fast pipelined implementations in both FPGA and ASIC [11-15]. However, their implementations use a lot of logic gates and consume a lot of power to archive the high performance. Therefore, the implementations are too costly for mobile wireless applications to operate on the resource-constraint wireless devices.

In this paper, the main focus lies on providing the efficient design of the security accelerator that consumes less power due to using fewer logic gates as well as satisfies enough throughputs that is required in IEEE 802.15.4 standard.

We propose the efficient architecture for ZigBee security accelerator with low cost and low power. Our design consists of the following modules: 1) compact AES core using one SBox; 2) AES-CCM module based on the mode toggling structure to reduce the number of logic gates and power consumption; 3) the efficient security material management module.

We have implemented the proposed design, which is targeted to Altera Stratix FPGA device family [16]. The design tools utilized in this work are ModelSim SE PLUS 5.5 [17] and QuartusII v6.0.

\section{AES DESIGN FOR LOW COST}

\section{A. Previous Works for AES Design}

Rijndael Algorithm [18] has been selected as the new Advanced Encryption Standard (AES) Algorithm by the National Institute of Standards and Technology (NIST). The AES is a symmetric block cipher algorithm and can process data blocks of 128 bits, using cipher keys with lengths of 128 , 192, and 256 bits. Its operations are performed in a certain number of rounds, which varies between 10, 12, and 14 depending on the size of key length. Because 128-bit key is used in CCMP of the security suites in IEEE 802.15.4, we consider AES design to support only 128-bit key length.

The AES algorithm has four basic transformations: SubBytes, ShiftRows, MixColumns, and AddRoundKey. The SubBytes transformation is a non-linear byte substitution that operates independently on each byte of the State using a substitution table (S-Box). The ShiftRows transformation is a cyclically shift operation with constant offsets, applied to the rows of the matrix. The MixColumns transformation operates on the State column-by-column, treating each column as a four-term polynomial. The AddRoundKey transformation performs XOR operation on the round key, which is obtained from the initial key by a key expansion procedure.

The AES algorithm consists of an initial transformation, followed by a main loop where nine iterations called rounds are executed. Each round transformation is composed of a sequence of four transformations mentioned above. For each round of the main loop, a round key is derived from the original key through a process called Key Scheduling. Finally, a last round consisting of three transformations SubBytes, ShiftRows, AddRoundKey is executed. Figure 1 shows the AES encryption process.

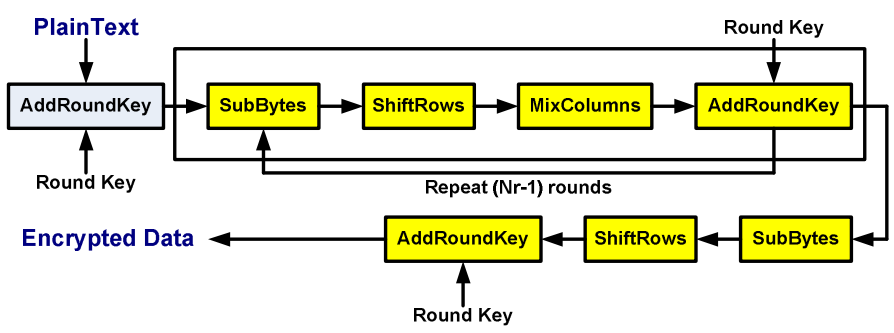

Figure 1. AES encryption process 


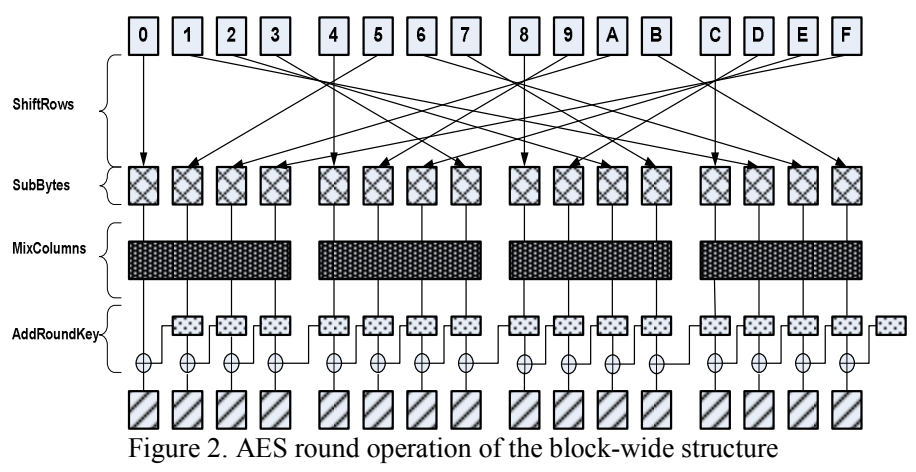

The AES algorithm takes the seed key and performs a key expansion routine to generate round keys which are used in the AddRoundKey transformation. In AES-128, total eleven 128bit round keys including a seed key are generated after Key Scheduling.

One of the most common AES implementation is called block-wide AES structure and uses a great deal of parallelism [19]. It mainly consists of 4 blocks: SubBytes/ShiftRows, MixColumns, Round Key Generator, and AES Control. Figure 2 illustrates the AES round operation of the block-wide structure.

In AES hardware implementation, AES S-Box design contributes a major role in optimization. When a block-wide AES encryption unit was design in VHDL, according to the synthesis result, area of S-Boxes respect to all the hardware in one round is $84.4 \%$ [20] and the total power consumption is at least $75 \%$ [21].

To reduce the power consumption and the logic usage in SubBytes block of the block-wide AES, various AES hardware implementation was introduced. We can figure out that 128-bit data block is divided into four 32-bit data blocks and each block is processed independently. Therefore, it is capable to implement AES module using only four AES S-Boxes and one 32-bit MixColumns to compact the AES implementation. By processing one round operation during four clock cycles with reusing four AES S-Boxes, we may reduce the resources by $75 \%$ ideally. Actually, the folded AES cannot cut the resources by $75 \%$ because of additional logics such as multiplexer, latches, and control logic.

Typical designs of the folded AES implemented by A. Satoh et al. [22]. This needs some additional logics such as a 128-bit register, a 96-bit register, a 32-bit $4 \times 1$ multiplexer, and control signal and logic comparing with the block-wide AES. The design results in [22] show that the folded design uses $50 \%$ of the resources required by the block-wide design, and has 4 times lower throughput.

We implement the folded AES which are similar to architecture in [22]. However, our folded AES has four AES SBoxes and those are also used in the key scheduling process. By doing so, we can reduce the 4 AES S-Boxes required in key scheduling process of the block-wide. However, one round operation takes 5 clock cycles, instead of four clock cycles in architecture in [22].

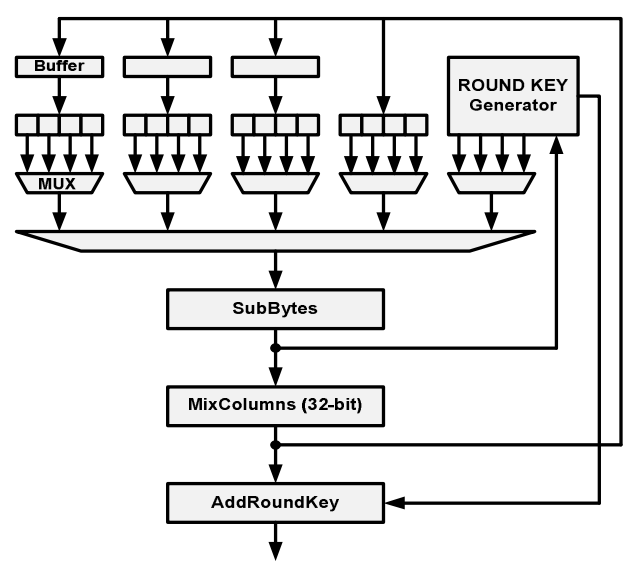

Figure 3. Architecture of the low cost AES core using only one AES S-Box

\section{B. Our AES Design for Low Cost}

To reduce the power consumption and hardware complexity, we introduce the low cost AES design with using only one AES S-Box. Figure 3 shows the block diagram of the low cost AES design. This design consists of three 32-bit registers, sixteen 8 -bit registers, five 4 by 1 multiplexers, one 5 by 1 multiplexers, a Round Key Generator, one 32-bit MixColumns, and AddRoundKey. During one AES round operation, it requires 20 SubBytes transformations: 16 times for AES encryption, 4 times for key scheduling.

In the block-wide, one round operation takes only one clock cycle with using 20 AES S-Boxes. In the low cost design, one round operation takes 20 clock cycles by reusing one AES SBox. For that reason, the data throughput in the low cost AES design is $1 / 20$ of the block-wide and $1 / 4$ of the folded design respectively. However, we can reduce the logic usage and the power consumption by reducing the AES S-Boxes which occupies much area.

TABLE I

SYNTHESIS AND SIMULATION RESULTS OF VARIOUS AES DESIGNS

\begin{tabular}{|l|r|r|r|}
\hline \multicolumn{1}{|c|}{ Measure } & Block-wide & Folded & \multicolumn{1}{c|}{$\begin{array}{c}\text { Our } \\
\text { design }\end{array}$} \\
\hline Device & \multicolumn{3}{|c|}{ Stratix EP1S10F484C5 } \\
\hline Logic elements & 691 & 692 & 702 \\
\hline $\begin{array}{l}\text { Memory bits } \\
\text { (Converted logic elements) }\end{array}$ & $\begin{array}{r}40,960 \\
(4,160)\end{array}$ & $\begin{array}{r}8,192 \\
(832)\end{array}$ & $\begin{array}{r}2,048 \\
(208)\end{array}$ \\
\hline Total Logic elements & 4,851 & 1,524 & 910 \\
\hline Clock cycles for one round & 1 & 5 & 20 \\
\hline Operating clock frequency (MHz) & 20 & 20 & 20 \\
\hline $\begin{array}{l}\text { Core dynamic thermal power } \\
\text { dissipation (mW) }\end{array}$ & 6.98 & 4.74 & 4.62 \\
\hline $\begin{array}{l}\text { Core static thermal power } \\
\text { dissipation (mW) }\end{array}$ & 9.19 & 2.89 & 1.72 \\
\hline Total thermal power dissipation & 16.17 & 7.63 & 6.34 \\
\hline
\end{tabular}

We demonstrate that the proposed AES core design occupies less area and consumes lower power than other designs from the synthesis and simulation results in Table 1 . The variance in logic element usage increases slightly more since the low cost AES design needs additional logics such multiplexers and 
latches instead of reducing memories and MixColumns. However, memory bits usage is $5 \%$ of the block-wide and $25 \%$ of the folded. To compare the total logic element usage of three kinds of AES design, we convert the memory bits usage to logic element usage. The total logic element usage of the low cost AES design is $18.76 \%$ of the block-wide and $59.71 \%$ of the folded. The static power consumption in the proposed AES core for IEEE 802.15 .4 is $16.9 \%$ of the block-wide and $59.5 \%$ of the folded design respectively. In addition, at the same operating clock frequency, the total power consumption in the proposed AES core is lower than others.

\section{SeCURITY ACCELERATOR ARCHITECTURE FOR IEEE 802.15 .4}

The overall architecture of the security accelerator is illustrated in Figure 4. The security accelerator has the following functionalities:

- Supporting all security suites: AES-CTR, AES-CBCMAC, AES-CCM

- ACL \& security materials management: ACL entry addition, deletion, search, and update

- Replay attack prevention: the validity check of the frame counter and the key sequence counter

- CBC-MAC countermeasure: the validity check of the MIC in AES-CBC-MAC and AES-CCM mode

It consists of seven main modules. An SMM is the security material management module. An MPDU_Parser_Constructor provides the functionalities relative to a MAC header and a security auxiliary header. In this paper, we define the security auxiliary header to a pair of a key sequence counter and a frame counter added to the MPDU payload while the security suite is either AES-CCM or AES-CTR. This MPDU_Parser_Constructor checks the size of 'Addressing fields' since it has variable length according to the addressing mode, i.e., either a short address or an extended address. It calculates the MAC header length according to the size of 'Addressing fields' in an MPDU. It contains a 28-bit length buffer to save the MAC header which is transmitted from the 802.15.4 MAC H/W over the MAC_I/F. On the decapsulation process, it also latches the security auxiliary header which is transmitted from the 802.15.4 MAC H/W over the MAC_I/F and delivers the latched security auxiliary header to the Cipher_Engine. A Controller contains a finite state machine to synchronize and orchestrate the whole process and control the information flow. A MemoryBuffer is composed of memory blocks and buffers to latch data.

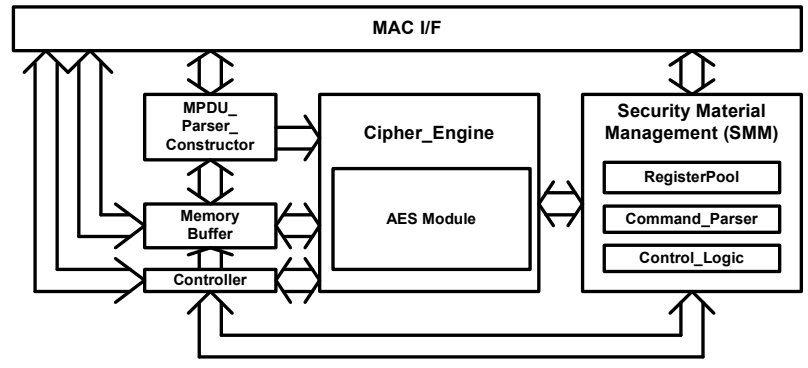

Figure 4. Overall security accelerator architecture

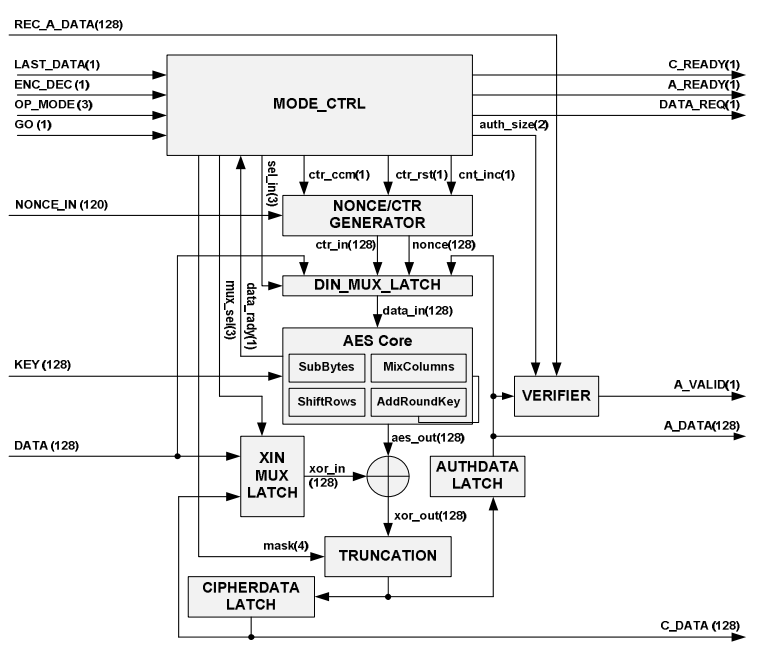

Figure 5. Block diagram of the cipher engine

\section{A. Design of Cipher Engine based on AES-CCM}

AES-CCM algorithm consists of two processes. One is MIC calculation with $\mathrm{CBC}-\mathrm{MAC}$ and the other is data encryption with counter mode. In AES-CCM, almost the same structure can be used to both MIC calculation and data encryption. Therefore, in general there are two methods in designing AESCCM module in hardware.

In the sequential structure, by controlling input and output data to or from an AES module according to the operating mode, it supports both counter and CBC-MAC mode with using only one AES module. In [24], AES-CCM is implemented in this way. However, its response time is directly proportional to the payload size. Therefore, in the applications which require short response time, the sequential structure should be operating at very fast clock frequency to satisfy the requirements [25].

In the parallel structure, an AES-CCM module uses two AES modules. One AES module is used for MIC calculation in CBC-MAC mode and the other is used for data encryption in counter mode. In [26, 27, 28], AES-CCM is implemented in the parallel structure. The response time are not dependent on the payload size in the parallel structured. However, it uses two AES modules so that it uses nearly 2 times more logic gates than the sequential structured. In addition, it also consumes more power than the sequential structured.

TABLE II

FEATURES OF THE THREE AES-CCM STRUCTURES: SEQUENTIAL, PARALLEL, AND MODE-TOGGLING

\begin{tabular}{|l|r|r|r|}
\hline \multicolumn{1}{|c|}{ Measure } & Sequential & Parallel & \multicolumn{1}{c|}{$\begin{array}{c}\text { Mode } \\
\text { toggling }\end{array}$} \\
\hline Device & \multicolumn{3}{|c|}{ Stratix EP1S10F484C5 } \\
\hline Logic elements & 1,053 & 1,695 & 1,276 \\
\hline $\begin{array}{l}\text { Memory bits } \\
\text { (Equivalent logic elements) }\end{array}$ & $\begin{array}{r}40,960 \\
(4,160)\end{array}$ & $\begin{array}{r}73,728 \\
(7,488)\end{array}$ & $\begin{array}{r}40,960 \\
(4,160)\end{array}$ \\
\hline Total logic elements & 5,213 & 9,183 & 5,436 \\
\hline Operating clock frequency & 5 & 5 & 5 \\
\hline Response time ( $\mu$ s) & 14.96 & 0.88 & 0.88 \\
\hline Throughput (Mbps) & 28 & 56 & 28 \\
\hline $\begin{array}{l}\text { Throughput / Logic usage } \\
\text { (Kbps / \#) }\end{array}$ & 5.70 & 6.27 & 5.37 \\
\hline
\end{tabular}


The mode-toggling structure for AES-CCM is introduced in [29]. We adopt the mode-toggling structure when implementing AES-CCM module for IEEE 802.15.4 to reduce the hardware complexity in ZigBee device. This structure calculates the MIC data and performs data encryption with one AES module by controlling input data to an AES module. It calculates the MIC data in CBC-MAC mode and performs data encryption in counter mode by turn every 128 -bit block data.

Table 2 summarizes the features of the three AES-CCM structures. By adopting mode-toggling structure in AES-CCM hardware implementation, we can reduce the logic usage as nearly a half as the parallel structure with having same response time as the parallel structure.

The AES_CCM based cipher engine for IEEE 802.15.4 has to provide all these operating modes (AES-CTR, AES-CBC$\mathrm{MAC}$, and AES-CCM). The architecture of our cipher engine is shown in Figure 5. It consists of 5 main blocks: AES Module, NONCE/CTR GENERATOR, MODE_CTRL, VERIFIER, and 4 latches. AES-CCM is the mode-toggling structure.

\section{B. Design of Security Material Management}

Before discussing about the SMM (Security Material Management) design, we first address general CAM (Content Addressable Memory) design methodologies. In our design, CAM is used for searching a cryptographic key that is appropriate to the target MAC address of incoming or outgoing data. CAMs have a different feature from standard memories simply by being content addressable. If a system only needs to know whether a matched item is located in a memory, CAMs have the ability of parallel search based on content across all stored items.

CAMs use different numbers of comparators depending on the performance requirements. Broadly, CAMs can be divided into two architectures: sequential and parallel. The sequential CAM uses only one comparator and one memory block. On the other hand, the parallel CAM uses several comparators and memory blocks.

In the sequential structured CAM, as the number of saved contents becomes larger, it takes more time to search the matched content. That is to say, if the matched content is located in the 100-th entry, we can confirm the result of a read operation after the CAM reads data from a MEMORY 100 times.

In the parallel structured CAM, the more pairs of comparator and memory, the earlier it takes time to confirm the result of read operation. Let $\mathrm{T}_{\text {search }}, \mathrm{Addr}_{\text {entry }}, \mathrm{t}_{\text {clock}}$, and $\mathrm{N}_{\text {mem }}$ denote the search time, a memory address of the matched entry, a clock period, and the number of memories, respectively.

$$
T_{\text {search }}=t_{\text {clock }} \times\left\lceil\frac{A d d r_{\text {entry }}}{N_{\text {mem }}}\right\rceil
$$

As a CAM uses more pairs of comparator and memory, it can have a shorter search time. Figure 6 shows the search time of several CAMs according as which of memory address the matched entry is located in, when the operating clock frequency is $50 \mathrm{MHz}$ and CAMs accommodate maximum 256 entries. When the matched data is presented in the last entry, the search time in the sequential structured CAM is $5.12 \mu \mathrm{s}$. However, the search time in a parallel structure CAM with 8 pairs of comparator and memory is only $0.64 \mu \mathrm{s}$.

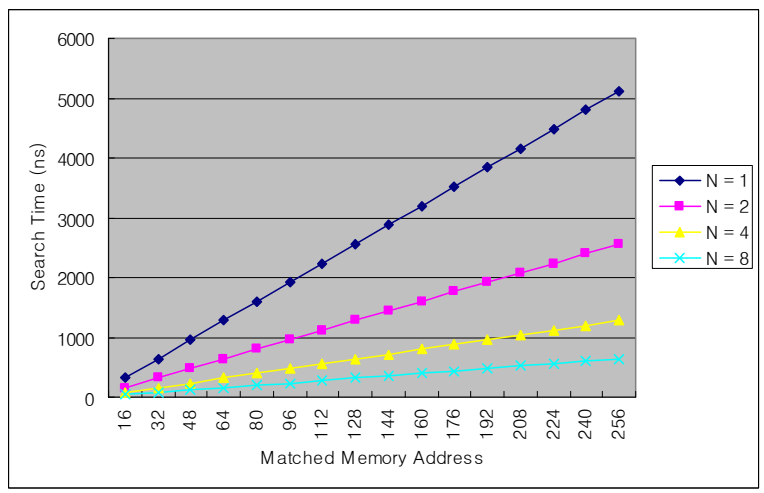

Figure 6. Search time in several CAM architectures

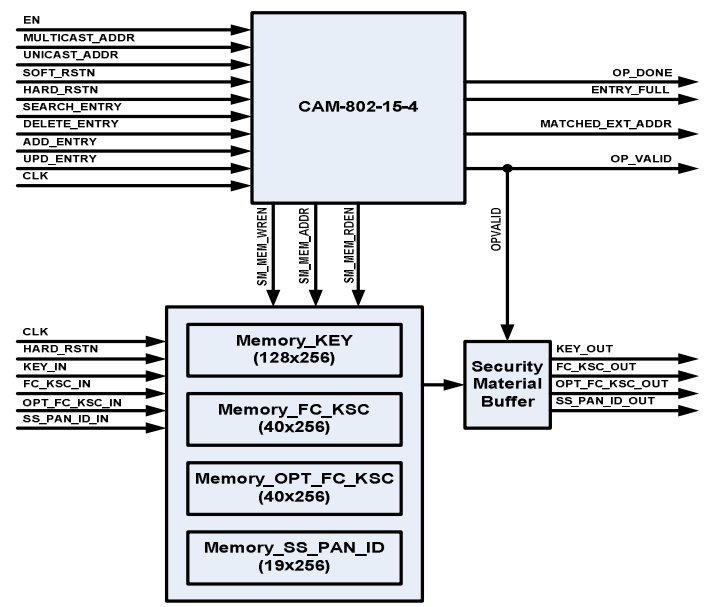

Figure 7. Block diagram of SMM

Figure 7 shows the block diagram of the implemented SMM for IEEE 802.15.4. It consists of three main blocks: a MEMORY, a CAM-802-15-4, and a SecurityMaterialBuffer. A MEMORY consists of 4 memory sub-blocks: a Memory_KEY, a Memory_FC_KSC, a Memory_OPT_FC_KSC, and a Memory_SS_PAN_ID. Four memory sub-blocks are just memory blocks. A Memory_KEY contains symmetric keys and a Memory_FC_KSC saves frame counters and key sequence counters. A Memory_OPT_FC_KSC contains optional external frame counters and optional external key sequence counters and a Memory_SS_PAN_ID contains security suites and PAN identifiers. A SecurityMaterialBuffer is a simple 227-bit length latch. A CAM-802-15-4 is the CAM for IEEE 802.15.4.

\section{RESULTS}

When we calculate the performance of our implemented security accelerator, we assume that the MAC payload size is 
96 bytes and the MIC size is 64 bits and the operating clock frequency is $3 \mathrm{MHz}$.

When the security suite is AES-CCM, the data throughput in security accelerator is more than $600 \mathrm{kbps}$ and it is 2.4 times more than the $250 \mathrm{kbps}$ which is the MAC data processing speed in IEEE 802.15.4 standard. The response time on AESCCM operation is $407 \mu \mathrm{s}$ including the search time in the SMM. This is less than $500 \mu$ s which is the critical response time as mentioned in Section 2. The search time in the SMM is less than $100 \mu \mathrm{s}$. This is less than the maximum allowable search time, $156.25 \mu \mathrm{s}$, as mentioned in Section 2. The verification delay on AES-CCM operation is $220 \mu \mathrm{s}$. This is less than the critical verification delay at $250 \mathrm{kbps}$ transmission speed in PHY, 250 $\mu$ s. Therefore the implemented security accelerator satisfies all of constraints required in IEEE 802.15.4 standard.

The total power consumption of the implemented security accelerator in FPGAs is about $29 \mathrm{~mW}$. The dynamic power of the implemented security accelerator occupies $7.6 \%$ of the total power consumption. It is necessary to ascertain whether the power consumption feature in security accelerator is acceptable in IEEE 802.15.4 application.

\section{CONCLUSIONS}

We presented efficient designs for ZigBee security accelerator. We used only one S-Box to design AES core to reduce the logic usage and power consumption. Besides, we used only one AES module and adopt the mode toggling approach to implement an efficient AES-CCM. We also presented the optimal security material module to reduce the logic usage and power consumption.

These design methodologies allows the cryptographic core to adopt lower clock frequency as slow as possible while still satisfying specification, so that logic usage is lower than pervious design. The energy consumption is also lower.

\section{ACKNOWLEDGMENT}

This research was supported by a grant(CR070019) from Seoul R\&BD Program funded by the Seoul Development Institute of Korean government.

\section{REFERENCES}

[1] Yang Xiao, Hsiao-Hwa Chen, Bo Sun, Ruhai Wang, Sakshi Sethi, "MAC Security and Security Overhead Analysis in the IEEE 802.15.4 Wireless Sensor Networks", EURASIP Journal on Wireless Communications and Networking, May 2006.

[2] Dandalis A., Prasanna V.K., Rolim J.D., "A Comparative Study of Performance of AES Final Candidates Using FPGAs", Cryptographic Hardware and Embedded Systems Workshop (CHES 2000), Worcester, Massachusetts, 2000.

[3] Elbirt A.J., Yip W., Chetwynd B., Paar C., "An FPGA Implementation and Performance Evaluation of the AES Block Cipher Candidate Algorithm Finalists", Third Advanced Encryption Standard (AES3) Candidate Conference, New York, 2000.

[4] Gaj K. and Chodowiec P., "Comparison of the hardware performance of the AES candidates using reconfigurable hardware", Third Advanced Encryption Standard (AES3) Candidate Conference, New York, 2000.
[5] Gaj K. and Chodowiec P., "Hardware performance of the AES finalistssurvey and analysis results", Technical Report, George Mason University, 2000, available at http://ece.gmu.edu/crypto/AES survey.pdf

[6] Ichikawa T. and Matsui T. "Hardware Evaluation of the AES Finalists", Third Advanced Encryption Standard (AES3) Candidate Conference, New York, 2000.

[7] Fischer V. and Drutarovsk'y M., "Two Methods of Rijndael Implementation in Reconfigurable Hardware", Cryptographic Hardware and Embedded Systems (CHES 2001), Paris, France, 2001.

[8] McLoone M. and McCanny J.V. "High Performance Single-Chip FPGA Rijndael Algorithm Implementations", Cryptographic Hardware and Embedded Systems (CHES 2001), Paris, France, 2001.

[9] McLoone M. and McCanny J.V., "Single-Chip FPGA Implementation of the Advanced Encryption Standard Algorithm", Field-Programmable Logic and Applications (FPL 2001), Belfast, Northern Ireland, UK, 2001

[10] McLoone W., McCanny J.V., "Rijndael FPGA implementation utilizing look-up tables", IEEE Workshop on Signal Processing Systems, 2001.

[11] Standaert F.X., Rouvroy G., Quisquater J.J., Legat J.D., "A methodology to implement block ciphers in reconfigurable hardware and its application to fast and compact AES RIJNDAEL", International Symposium on Field-Programmable Gate Arrays (FPGA 2003), Monterey, CA, 2003.

[12] Chodowiec P., Khuon P., Gaj K., "Fast implementations of secret-key block ciphers using mixed inner- and outer-round pipelining", International Symposium on Field-Programmable Gate Arrays (FPGA 2001), Monterey, CA, 2001

[13] Verbauwhede I., Schaumont P., Kuo H., "Design and performance testing of a 2.29-GB/s rijndael processor", IEEE Journal of Solid-State Circuits, Volume: 38 Issue: 3, March 2003.

[14] Lin T.F., Su C.P., Huang xC.T., Wu C.W., "A high-throughput low-cost AES cipher chip", IEEE Asia-Pacific Conference on ASIC, 2002.

[15] Lutz A.K., Treichler J., G"urkaynak F.K., Kaeslin H., Basler G., Erni A., Reichmuth S., Rommens P., Oetiker S., Fichtner W., "2Gbit/s Hardware Realizations of RIJNDAEL and SERPENT: A Comparative Analysis", Cryptographic Hardware and Embedded Systems (CHES 2002), San Francisco Bay, CA, 2002.

[16] Altera website, http://www.altera.com, 2006

[17] Mentor Graphics website, http://www.model.com, 2006

[18] J. Daemen and V. Rijmen, "AES Proposal: Rijndael, AES algorithm submission", September 3, 1999.

[19] Panu Hämäläinen, Marko Hännikäinen, Timo D. Hämäläinen, "Efficient Hardware Implementation of Security Processing for IEEE 802.15.4 Wireless Networks", ISSA 2005.

[20] Song J. Park, "Analysis of AES hardware Implementations", available at http://islab.oregonstate.edu/koc/ece679/project/2003/park.pdf

[21] Morioka S. and Satoh A. "An Optimized S-Box Circuit Architecture for Low Power AES Design", Cryptographic Hardware and Embedded Systems (CHES 2002), San Francisco Bay, CA, 2002.

[22] Satoh A., Morioka S., Takano K., Munetoh S., "A Compact Rijndael Hardware Architecture with S-Box Optimization", Theory and Application of Cryptology and Information Security (ASIACRYPT 2001), Gold Coast, Australia, 2001

[23] IEEE standard 802.15.4, 2006.

[24] Arshad Aziz, Nassar Ikram, "Hardware Implementation of AES-CCM For Robust Secure Wireless Network", at available http://icsa.cs.up.ac.za/issa/2005/Proceedings/Research/069_Article.pdf

[25] Duhyun Bae, Gwanyeon Kim, Jiho Kim, Sehyun Park, Ohyoung Song, "An Efficient Design of CCMP for Robust Security Network", LNCS 3935, July 2006.

[26] Emmanuel López-Trejo, Francisco Rodríguez-Henríquez, and Arturo Díaz-Pérez, "An Efficient FPGA implementation of CCM mode using AES”, LNCS 3935, July 2006.

[27] Duhyun Bae, Gwanyeon Kim, Jiho Kim, Sehyun Park, Ohyoung Song, "Design and Implementation of Efficient Cipher Engine for IEEE 802.11i Compatible with IEEE 802.11n and IEEE 802.11e", LNCS 3802, December 2005.

[28] Duhyun Bae, Jiho Kim, Sehyun Park, Ohyoung Song, "Design and Implementation of IEEE 802.11i Architecture for Next Generation WLAN", LNCS 3822, December 2005.

[29] Duhyun Bae, Gwanyeon Kim, Jiho Kim, Sehyun Park, Ohyoung Song, "An Efficient Design of CCMP for Robust Security Network", LNCS 3935, July 2006. 